\title{
Structure of pauses in speech in the context of speaker verification and classification of speech type
}

\author{
Magdalena Igras-Cybulska ${ }^{1 *}$, Bartosz Ziółko ${ }^{1,2}$, Piotr Żelasko ${ }^{1,2}$ and Marcin Witkowski ${ }^{1,2}$
}

\begin{abstract}
Statistics of pauses appearing in Polish as a potential source of biometry information for automatic speaker recognition were described. The usage of three main types of acoustic pauses (silent, filled and breath pauses) and syntactic pauses (punctuation marks in speech transcripts) was investigated quantitatively in three types of spontaneous speech (presentations, simultaneous interpretation and radio interviews) and read speech (audio books). Selected parameters of pauses extracted for each speaker separately or for speaker groups were examined statistically to verify usefulness of information on pauses for speaker recognition and speaker profile estimation. Quantity and duration of filled pauses, audible breaths, and correlation between the temporal structure of speech and the syntax structure of the spoken language were the features which characterize speakers most. The experiment of using pauses in speaker biometry system (using Universal Background Model and i-vectors) resulted in $30 \%$ equal error rate. Including pause-related features to the baseline Mel-frequency cepstral coefficient system has not significantly improved its performance. In the experiment with automatic recognition of three types of spontaneous speech, we achieved $78 \%$ accuracy, using GMM classifier. Silent pause-related features allowed distinguishing between read and spontaneous speech by extreme gradient boosting with $75 \%$ accuracy.
\end{abstract}

Keywords: Pauses, Fillers, Punctuation, Polish, Biometry, Spontaneous speech, Speech classification

\section{Introduction}

A set of common disfluencies interferes with discourse consistency in spontaneous speech. The most important ones are filled pauses, restarts, changes of syntax during the utterance, and inclusions of intervening sentences. Within words, the most frequent disfluencies are repetitions, repairs and prolongations of conjunctives, prepositions, and final syllables. As far as human perception can focus on the meaning of the utterance and extract the desired information, an automatic speech recognition system literally recognizes whole acoustic content of the speech signal. As a result, the transcription is redundant with notation of disfluencies or slips of the tongue but diminished of the other types of information present in a signal, like punctuation. Pause detection is usually used

\footnotetext{
* Correspondence: migras@agh.edu.pl; http://www.dsp.agh.edu.pl; http://techmo.pl

${ }^{1}$ Department of Computer Science, Electronics and Telecommunications, AGH University of Science and Technology, Al. Adama Mickiewicza 30, 30-059 Kraków, Poland

Full list of author information is available at the end of the article
}

only to extract voice activity regions for further processing or to remove undesirable disfluencies. On the other hand, the information on pauses properties patterns can significantly enrich high-level information of speech signal. In recent years, analysis of multi-layered linguistic and paralinguistic metadata of recordings received focused attention [1].

We assume that the pauses properties in speech signal are strongly individualized between speakers and influenced by situational context and cognitive task. This study aims to verify if the information on pauses can be useful for speaker biometry systems (experiment 1) and for recognition of different types of spontaneous speech (experiment 2) as well as distinguishing between read and spontaneous speech (experiment 3).

The information is meaningful for creating a speaker psycho-social profile. Additionally, it helps in discourse analysis for different kinds of situational context or linguistic task [27]. The types of speech differ in situational context, the task involving a cognitive load as well as 
level of spontaneity and have direct impact on speech fluency. For the first type, presentations prepared on a given subject represent typical informative speech in a formal situation. For the second type, oral translations performed by professional interpreters are partly imitative against the original speech. Simultaneity of listening and speaking engages complex cognitive functions. For the third type, radio interviews represents spontaneous speech extracted from dialogues with slow turn-taking, mainly storytelling (indicating more informal situation). Although the pauses in each type of speech were characterized in numerous analyses (e.g., [33-35]), there has been a lack of automatic classification of speech type based on silent pause-related cues only. In a similar study dealing with three classes of spontaneous speech [32], a comparable accuracy was obtained, though much more features was utilized.

Research on the recognition of read and spontaneous speech can have an impact in the field of automatic assessment of speaker preparation for the task, and elocution abilities. In this application, a feedback on similarity to fluent read speech would help speaker improve their level of oratorical skills.

Modeling of pauses in spoken language can be also applied to a more natural-sounding speech synthesis systems. The impact of pauses analysis for speech technology is particularly important for spontaneous speech recognition, which remains a challenging task [28]. Some results of presented works have already been used for building pauses models for automatic speech recognition system which is developed in AGH University and Techmo [29].

The paper is organized as follows: in the rest of Section 1, the background of the pauses appearance and role in speech is presented and the state-of-theart of speaker recognition systems is briefly discussed. Next, the collected database is described in Section 2. In Section 3, we summarize adopted method of database processing, features extraction and statistical tools. Section 4 contains results of our experiments, which are discussed in Section 5. The paper is concluded in Section 6.

\subsection{Pauses}

The research shows three types of acoustic pauses in spoken language. The most intuitive is silent pauses (s_p), as regions of signal where no voice activity is recorded.

The second types are filled pauses (f_p)-pseudowords-that do not affect sentence meaning, like $y y y$, eee, $h m m, m m m, y m, y h$ (in SAMPA notation: III, eee, $x m m, m m m, I m, I x)$ but perturb utterance fluency. The sounds of filled pauses are specific for language (in Polish, the most common are $y y y / y h$ and $m m m$, while for English-um) and specific for speaker's habits. They can appear even 10-20 per minute in case of inexperienced speakers.

The third sort of pauses that we consider are breath pauses (b_p). In case of normal physiological condition, the value of breath per minute is $12-20$ while resting, and as prior work showed, it is 10-12 during speech production [2].

Considering the origin of the pause usage we marked out: (1) regular natural pauses caused by respiration activity (breath pauses), (2) irregular intentional pauses, purposely used as a stylistic form, especially by professional speakers (silent pauses), and (3) irregular, unintentional disfluencies, effects of uncertainty, hesitations or short reflections (acoustic events like silent pauses or filled pauses).

\subsection{Pauses vs. paralinguistic information}

Depending on the speaker and situational/social context, pauses may be characterized by different properties. One of them is a type of personality of the speaker and his speaking habits. Another important factor is speaker preparation for the task, level of oratorical skills, and elocution abilities. Durations of pauses depend also on the kind of linguistic task. One can easily assume that stress during speaking is an important factor dictating the frequency and lengths of pauses. Pauses can be also considered in the terms of performative aspects of speech. Filled pauses, among other disfluencies, were successfully used for recognition of three levels of spontaneity and applied to speaker role recognition with over $70 \%$ precision [32]. Pauses were also described as traces of cognitive activity or a mirror of cognitive processes. In the situation of simultaneous interpretation, they were studied in [33] and [34].

Pause duration was reported to correlate with social attributes of speaker, even ones such as region, ethnicity, age, and gender [3]. Cross-cultural study of silent pauses in selected European languages (Polish was not included) revealed differences in pause durations between languages [4], but their distribution is usually similar and can be well estimated by bi-Gaussian model [5].

Some medical aspects of different types of pauses were investigated in context of affective state [6] and physical [7] or mental [8] condition of the speaker, e.g. schizophrenics make pauses around $10 \%$ more often, which are also around $10 \%$ longer [8].

In speech technology, information on pauses is used in majority of algorithms of automatic punctuation detection $[9,10]$. It has been shown that $95 \%$ of silent pauses longer than $350 \mathrm{~ms}$ are the sentence boundaries [11].

Pausing behavior in speech, although conditioned by articulatory processes, was proved to be partly related to cognitive processes [12]. It implies that it can be 
changed by learning. Nevertheless, the fact was proved only for the grammatical pauses, while for the ungrammatical ones the opposite was observed.

\subsection{Speaker recognition}

Speaker recognition is the process of analysis of the speaker identity based on voice characteristics. The main tasks of a speaker recognition system include verification and/or identification. The aim of identification is to choose one of many speakers based on a speech signal, whereas verification is the process of determination whether assigned speaker was chosen correctly. In line with the particular usage specification, those systems may be divided into text-dependent or text-independent. A text-dependent system assumes that recognition process is based on a specific fixed phrase, i.e., each analyzed recording contains the same sentence. In textindependent scenario, speakers may be identified or verified by a random utterance [13]. The second system is more challenging, since it is much more complicated due to phonetically mismatched voice samples in training and recognition phases.

Automatic speaker recognition systems consist of two main functionalities-enrollment and verification. During enrollment phase, voiceprint or model of a speaker is calculated based on extracted features from voice samples. Verification is based on comparison of processed input speech signal against the speaker model enrolled previously. There are many discriminative features that may be used to distinguish a speaker. Lowlevel features, like formants or energy, contain information connected with voice generation. Mel-frequency cepstral coefficients (MFCCs) are used most frequently to parameterize voice signals $[14,21,24]$.

\subsection{Pause-related features in speaker recognition task}

In past several years, there has been an observable tendency to include prosodic features to speaker recognition problem. High-level features are associated with linguistic and behavioral characteristics of each speaker [15]. In majority of approaches, features related to pitch, energy, and segmental duration were investigated [16-18] and by including these parameters, the system accuracy increased by about $10 \%$. Peskin et al. [16] experimented also with pause duration and frequency and found out that pause-related feature set was the least significant compared to other groups of prosodic features. In Sönmez et al. [17] experiment, pause duration was modeled with shifted exponential and together with voiced segments duration gave $3.5 \%$ improvement in speaker recognition task. It was also proved that patterns of pauses in network traffic introduced when encoding an audio signal are speaker-specific, and that they are sufficient to weaken the anonymity of the speaker in encrypted voice communication [19].

Therefore, the goal of this research is to check if pauses may be used as one of the high-level factors and potentially improve existing systems. To the best of authors' knowledge, the pauses features were not yet directly used in any speaker recognition system, in particular, in any Polish one.

\section{Materials}

The prepared corpus of spontaneous Polish speech consisted of different types of monologues in formal or semi-formal situations. Total duration of recordings is $120 \mathrm{~min}$, including utterances of 30 speakers (16 male, 14 female). Among them, there are both experienced or professional speakers (politicians, professors, professional translators, radio interviewees) and inexperienced speakers (students) [38].

The first group of recordings $(30 \mathrm{~min}$ ) is formed by utterances from orations or public presentations: speeches and reports from European Parliament [20], sessions of a faculty council, students' lectures, and reviews. All the speeches, although preceded by preparation of the speakers or supported by slides, were not read and are characterized by all the typical features of spontaneous speech.

The second part of the corpus (30 min) consisted of recordings of real-time translation of orations during European Parliament sessions [20]. This sort of utterances is specific kind of spontaneous speech, where the speech rate of the translator is determined by the style of the speaker being translated. However, they are situations of formularization of own utterance, which causes their spontaneous character and induces presence of imperfections specific for spontaneous speech.

The third type of recordings $(60 \mathrm{~min})$ is radio broadcasts, which were prepared by removing the voice of interviewer, leaving only the expressions of interviewees. The length of recordings after preparation was $10 \mathrm{~min}$ for each speaker (three females and three males).

Another corpus of read speech was prepared, for comparison with spontaneous speech and for evaluation tests. It consisted of recordings from audio books and AGH Audio-Visual Speech Database (50 speakers, $15 \mathrm{~min}$ of continuous speech for each speaker).

Since the recordings originate from different sources, they vary in quality, type, and level of background noise and SNR factor. Diversified conditions of recording (equipment, environment, transmission channel variability, and distance from the speaker to microphone) determined whether the signal contained events of our interest (e.g., recordings with low SNR or too big distance from mouth to microphone do not contain information on breath pauses). 
The recordings were labeled with $\mathrm{P}$ for presentations/ orations $\mathrm{T}$ for translations, $\mathrm{R}$ for radio dialogues, and $\mathrm{A}$ for audiobooks and other sources of read speech and described with number of the speaker and duration of utterance (in minutes).

\section{Methods}

\subsection{Pauses tagging and annotation}

First we transcribed orthographically the content of the recordings to clean (skipping disfluencies, filled pauses or repairs) and syntactically correct texts. On the basis of the observation of the process, the factors affecting the imprecision and ambiguity of inserting punctuation in the transcripts were collected. One of the impediments was ambiguous intonation, especially in case of inexperienced speakers. It manifested as "enumerating" tone of voice, which caused the speaker to preserve the same tone during commas and full stops. Another symptom was construction of multiple complex sentences with every clause starting with a conjunctive pronounced with extended phonation. In such cases, the decision of inserting comma or full stop remained subjective. When a speaker did not signalize the phrases and sentences border with their pronunciation, intonation or pauses, the punctuation was based on the meaning of the utterance. The last word of preceding sentence was often bonded with the first in

Table 1 Explanation of adopted abbreviations

\begin{tabular}{|c|c|c|c|}
\hline Parameter & Description & Parameter & Description \\
\hline S_p & Silent pause & $f_{29}$ & f_p(m) per minute \\
\hline f_p $(y)$ & Filled pause "yyy" & $f_{30}$ & $\%$ of $f \_p(m)$ time in recording \\
\hline f_p $(m)$ & Filled pause "mmm" & $f_{31}$ & mean f_p(m) duration [ms] \\
\hline b_p & Breath pause & $f_{32}$ & f_p(m) duration std. dev. [ms] \\
\hline$f_{1}$ & s_p $\geq 100$ ms per minute & $f_{33}$ & f_p(m) duration .25 quantile [ms] \\
\hline$f_{2}$ & $\%$ of s_p time in recording & $f_{34}$ & f_p(m) duration median [ms] \\
\hline$f_{3}$ & mean s_p duration [ms] & $f_{35}$ & f_p(m) duration .75 quantile [ms] \\
\hline $\mathrm{f}_{4}$ & s_p duration std. dev. [ms] & $f_{36}$ & b_p per minute \\
\hline$f_{5}$ & s_p duration .25 quantile [ms] & $f_{37}$ & $\%$ of b_p time in recording \\
\hline$f_{6}$ & s_p duration median [ms] & $f_{38}$ & mean b_p duration [ms] \\
\hline$f_{7}$ & s_p duration .75 quantile [ms] & $f_{39}$ & b_p duration std. dev. [ms] \\
\hline$f_{8}$ & s_p $\geq 150$ ms per minute & $f_{40}$ & b_p duration .25 quantile [ms] \\
\hline$f_{9}$ & $\%$ of s_p time in recording & $f_{41}$ & b_p duration median [ms] \\
\hline$f_{10}$ & mean s_p duration [ms] & $\mathrm{f}_{42}$ & b_p duration .75 quantile [ms] \\
\hline$f_{11}$ & s_p duration std. dev. [ms] & $f_{43}$ & \#w/min - number of words per minute \\
\hline$f_{12}$ & s_p duration .25 quantile [ms] & $f_{44}$ & \#./min - number of sentence per minute \\
\hline$f_{13}$ & s_p duration median [ms] & $\mathrm{f}_{45}$ & Mean sentence duration [s] \\
\hline$f_{14}$ & s_p duration .75 quantile [ms] & $f_{46}$ & \#w/sent - number of words in sentence \\
\hline$f_{15}$ & s_p $\geq 200$ ms per minute & $f_{47}$ & \#,/sent - number of phrases per minute \\
\hline$f_{16}$ & $\%$ of s_p time in recording & $\mathrm{f}_{48}$ & Mean phrase duration [s] \\
\hline$f_{17}$ & mean s_p duration [ms] & $\mathrm{f}_{49}$ & \#w/phr - number of words per phrase \\
\hline$f_{18}$ & s_p duration std. dev. [ms] & $f_{50}$ & $\%$ of full stops correlated with n_p \\
\hline$f_{19}$ & s_p duration .25 quantile [ms] & $f_{51}$ & $\%$ of full stops correlated with s_p \\
\hline$f_{20}$ & s_p duration median [ms] & $f_{52}$ & $\%$ of full stops correlated with f_p \\
\hline$f_{21}$ & s_p duration .75 quantile [ms] & $f_{53}$ & $\%$ of full stops correlated with b_p \\
\hline$f_{22}$ & f_p(y) per minute & $f_{54}$ & $\%$ of commas correlated with n_p \\
\hline$f_{23}$ & $\%$ of $f_{-} p(y)$ time in recording & $f_{55}$ & $\%$ of commas correlated with s_p \\
\hline$f_{24}$ & mean f_p(y) duration [ms] & $f_{56}$ & $\%$ of commas correlated with f_p \\
\hline$f_{25}$ & f_p(y) duration std. dev. [ms] & $f_{57}$ & $\%$ of commas correlated with b_p \\
\hline$f_{26}$ & f_p(y) duration .25 quantile [ms] & & \\
\hline$f_{27}$ & f_p(y) duration median [ms] & & \\
\hline $\mathrm{f}_{28}$ & f_p(y) duration .75 quantile [ms] & & \\
\hline
\end{tabular}


the next one. In translators group we usually observed specific disorder of phonotactics involving artificial prolongations of whole words. Transposals of functional elements of sentences and reorganization of the sentence were also frequent events. It is common for inexperienced speakers to place intervening sentences during the speech or abusing certain words like let's say, just, simply (language-specific conversational fillers/discourse makers).

For each transcription, the number of words, full stops, and commas were counted. Then, the statistics of sentences and phrases lengths were computed: mean length of a sentence and a phrase, as well as a mean number of words in sentences and phrases. Then, in the places of punctuations signs, occurrences of pauses were verified. When a full stop was signalized by a silent pause, the time was tagged as s_p. (similarly for commas - s_p,), filled pause - f_p. (commas f_p,), b_p. for a breath pause (b_p, for commas). When no type of pause appeared, the place was tagged as n_p. (n_p,). The parameters were included in the feature vectors (Table 1). Time annotation of breaths and filled pauses were prepared manually with half-automated Annotator software. As a result, Master Label Files (mlf, HTK standard) were attached to each recording.

\subsection{Feature extraction}

In order to find silent pauses in all recordings, an ITU$\mathrm{T}$ G.729b compliant voice activity detector (VAD) was used, which relies on full band energy, low band energy, zero-crossing rate and a spectral measure to decide whether a 10-ms segment contains voice. Silent pauses were detected with different lower thresholds: 100, 150, and $200 \mathrm{~ms}$.

For each speaker, the amount and duration of each type of pauses were used to calculate number of pauses per minute, percentage of pause duration in the recording, and mean pause duration with its standard deviation as well as its median and quartiles. The parameters are listed and explained in Table 1.

\subsection{Statistical methods}

Values of extracted features $f_{n}$ (where $n=1, \ldots, P$ ) were standardized according to the equation

$$
\overline{f_{n, s}}=\frac{f_{n, s}-\mu\left(f_{n}\right)}{\sigma\left(f_{n}\right)},
$$

where $\overline{f_{n, s}}$ is a normalized value of the feature $f_{n}$ for $s$ th speaker, $\mu$ and $\sigma$ are mean and standard deviation of the variable $f_{n}$ in the examined population.

The properties of speaker $s$ are specified by vector $p_{s}$ $=f_{1, s} f_{2, s}, \ldots, f_{P, s}$ of length $P$, and its distance to mean value vector $p$ is computed as

$$
\gamma_{s}=\frac{1}{P} \sum_{n=1}^{P}\left|\overline{f_{n, s}}\right|
$$

In order to investigate potential correlation between speakers and parameters describing pauses in their speech, we computed correlation matrix

$$
\text { Corr }=\sum_{s=1}^{N}\left(p-p_{s}\right)\left(p-p_{s}\right)^{T}
$$

where $p$ is an average vector for all speakers, and $p_{s}$ is a vector that characterizes sth speaker. In the experiment number of speakers $N$ is equal to 30 . We performed the operation for $P=57$ parameters listed in Table 1.

For the parameters which could be obtained for every speaker in our corpus, (i.e., b_p and f_p(y) durations), we concluded an analysis of variance (ANOVA) in order to check the statistical significance of differences between speakers based upon only one of these parameters at a

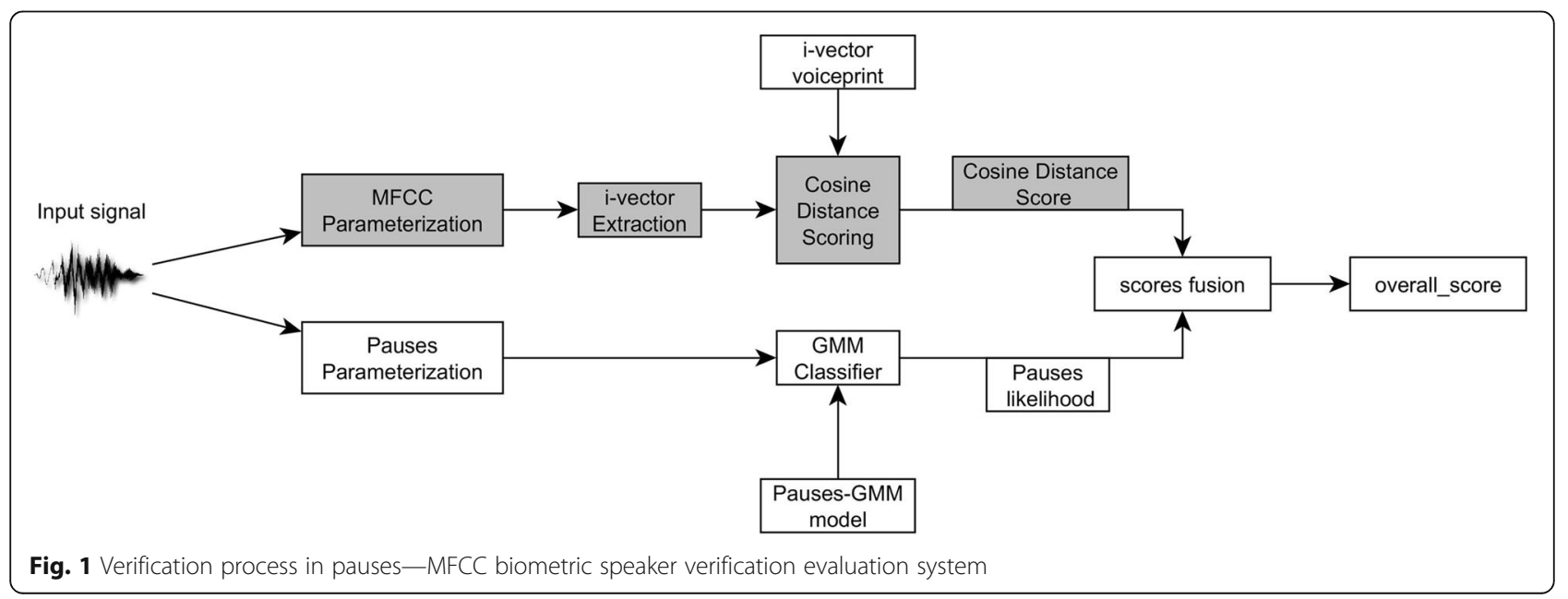


Table 2 Frequency of punctuation in transcripts: mean (standard deviation)

\begin{tabular}{llllllll}
\hline Recordings & $\begin{array}{l}\text { Words per } \\
\text { minute }\end{array}$ & $\begin{array}{l}\text { Sentences per } \\
\text { minute }\end{array}$ & $\begin{array}{l}\text { Sentence duration } \\
{[s]}\end{array}$ & $\begin{array}{l}\text { Words per } \\
\text { sentence }\end{array}$ & $\begin{array}{l}\text { Phrases per } \\
\text { minute }\end{array}$ & $\begin{array}{l}\text { Phrase duration } \\
{[\mathrm{s}]}\end{array}$ & $\begin{array}{l}\text { Words per } \\
\text { phrase }\end{array}$ \\
\hline Type P & $11,9(24,3)$ & $6,2(2,4)$ & $10,9(3,7)$ & $19,6(5,8)$ & $19,4(6,0)$ & $3,3(0,8)$ & $4,4(1,0)$ \\
Type T & $117,2(20,1)$ & $6,6(2,0)$ & $10,0(3,2)$ & $19,1(4,7)$ & $14,6(3,3)$ & $4,3(1,0)$ & $4,6(0,8)$ \\
Type R & $129,7(29,1)$ & $9,9(3,3)$ & $6,7(2,3)$ & $13,9(3,3)$ & $16,1(4,2)$ & $2,5(0,8)$ & $5,4(0,8)$ \\
All & $117,6(23,8)$ & $7,1(2,8)$ & $9,7(3,5)$ & $18,2(5,3)$ & $16,8(5,1)$ & $3,5(1,1)$ & $4,7(0,9)$ \\
speakers & & & & & & & \\
\hline
\end{tabular}

time. For clustering experiment, we used dendrogram method based on Euclidean metrics.

\subsection{Evaluation methods}

The scope of the first experiment was to verify if information on pauses can enhance a biometric verification system, therefore an i-vector based system was set up as a baseline to perform evaluation process. The i-vector approach assumes creation of a Universal Background Model (UBM) with a vast amount of data during setup phase. This process is performed by maximum likelihood estimation of a Gaussian Mixture Model (GMM), using an Expectation Maximization (EM) algorithm with K-Means initialization. The next stage of i-vector modeling is transition from the GMM supervector space into a low dimensional subspace, which is able to represent a whole utterance as a vector of coordinates, called the ivector. To that end, a transformation matrix, called Total Variability matrix (TV), is estimated also using a maximum likelihood algorithm [30].

The aim of UBM is to represent common characteristics of all possible speakers, and the role of dimensionality reducing transformation is to select only the relevant ones for a given speaker. In enrollment process in the baseline system, the recording is first segmented to 20-ms frames and parameterized by Mel-frequency cepstral coefficients (MFCC), followed by feature warping [22]. Then, model of each system user is acquired by calculating frame posteriors using GMM-UBM and extracting i-vectors with a variational Bayes algorithm [30]. The enrolled model represents unique biometrical features of a given speaker. The Pauses system models each user by extracting prosodic features from a larger recording segment, and thus requires at least $1 \mathrm{~min}$ of audio for enrollment and verification.

The final system is acquired by combining the final scores of i-vector/MFCC and Pauses systems in evaluation process. Because of that, parameterization based on pauses was added as a parallel to i-vector system. General verification method is presented in Fig. 1. MFCC features extracted from an input signal are forwarded into i-vector extractor. Cosine distance scoring

$$
\operatorname{CDS}(w 1, w 2)=\frac{w 1 * w 2}{\|w 1\|\|w 2\|},
$$

where $w 1$ and $w 2$ are $\mathrm{i}$-vectors, was used to obtain a likelihood measure between verified and tested i-vector.

The final score of combined systems is computed with the Bosaris Toolkit [31].

Speaker verification system is a binary classifier, since it determines whether analyzed signal is or is not produced by user related to model stored in a system database. The output of the system is the information that user is a target (the analyzed model is the user model) or an impostor (the model was created in other user enrollment process). Evaluation is therefore based on analysis of target and impostor likelihood distributions. The more separated are those sets the better system works, since it is easier to choose a threshold that divides those sets. In general, expected value of target distribution is greater than the impostor one. Basing on likelihood distributions, it is possible to calculate cumulative distribution functions for targets and impostors. Those functions may be used to determine false positive ratio (FPR) and false negative ratio (FNR) functions which determine respectively the probability that an impostor is classified as a target and a target is classified as an impostor for a particular likelihood. Decision about particular threshold i.e. choice of operating point, is dependent on the use case of such system. Increasing the threshold results in lower FPR, but also means that more targets verification will fail. The value where FPR = FNR is called equal error rate (EER) and is widely used to determine performance of a verification system as a single

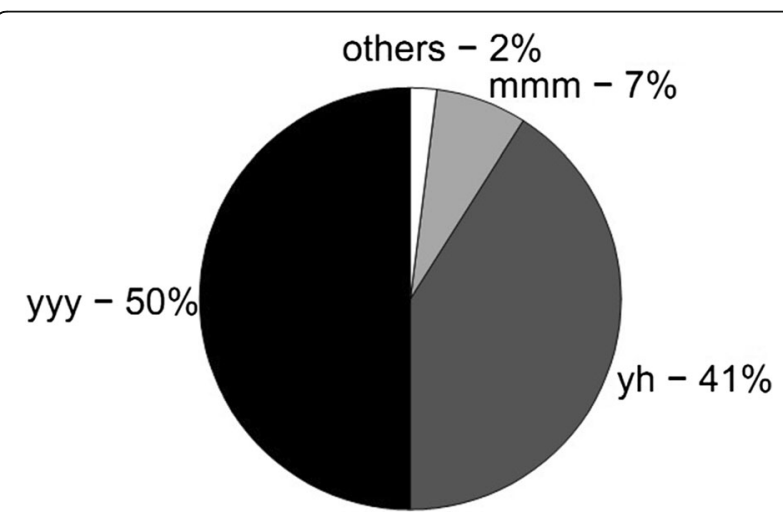

Fig. 2 Different types of filled pauses and frequency of their occurrence in $1 \mathrm{~h}$ corpus [23] 
Table 3 Frequency of silent, breath, and filled pauses in recordings: mean (standard deviation)

\begin{tabular}{lllllll}
\hline Recordings & $\#\left(s \_p>100 \mathrm{~ms}\right) / \mathrm{min}$ & \#(s_p > 150 ms)/min & \#(s_p > 200 ms)/min & \#f_p(yyy)/min & \#f_p $(\mathrm{mmm}) / \mathrm{min}$ & \#b_p/min \\
\hline Type P & $10,7(7,5)$ & $7,7(6,1)$ & $5,3(4,7)$ & $6,5(5,5)$ & $0,6(0,9)$ & $10,5(3,9)$ \\
Type T & $2,2(2,4)$ & $1,1(1,1)$ & $0,7(0,9)$ & $5,8(3,5)$ & $0,9(1,0)$ & $11,0(3,8)$ \\
Type R & $2,6(3,9)$ & $1,1(1,8)$ & $0,5(0,9)$ & $7,4(3,6)$ & $1,27(1,14)$ & $11,0(1,7)$ \\
All speakers & $5,7(6,6)$ & $3,7(5,0)$ & $2,5(3,7)$ & $6,4(4,3)$ & $0,9(1,0)$ & $10,8(3,4)$ \\
\hline
\end{tabular}

parameter. Other operating points of a system, where FNR is not equal to FPR are commonly evaluated with detection error trade-off (DET) plots [39], which include miss probability (FNR) at vertical axis and false alarm probability (FPR) at horizontal one.

In the second experiment, we verified if pause-related features are useful for automatic classification of three types of spontaneous speech-types P, T, and R. Classification is performed with 3-components GMMs. We applied leaveone-out cross-validation where each time one speaker's recording was the testing one and all the others formed the training set. The goal of the third experiment was to automatically distinguish two classes: read (50 audio books) and spontaneous speech (27 recordings of types $\mathrm{P}, \mathrm{T}$ and $\mathrm{R}$ ). Several classifiers were tested: decision tree, logistic regression, support vector machine (SVM), random forest classifier and Extreme Gradient Boosting (XGBoost) [36], using Scikit-learn toolkit [37]. Again, a leave-one-out cross-validation was applied.

\section{Results}

\subsection{Overall analysis of pauses appearance in speech}

Speech rate in spontaneous monologues is about 117 words per minute (with standard deviation between speakers is about 24 words/min). Mean length of a sentence (containing average 18 words) was about $10 \mathrm{~s}$, while mean length of a speech unit divided by punctuation (average 5 words) - $3.5 \mathrm{~s}$. The results were similar for both orations/ presentations, real time translations and interviews (more results are presented in Table 2).

The most commonly used types of filled pauses are: prolonged "yyy" (50\%), short "yh" (41 \%) and "mmm" (7 \% of counts) (see Fig. 2). For the purpose of this research, we grouped together the "yyy" and "yh" categories and skipped other fillers, which are very rare (2\%).

As for acoustically registered breath pauses, average for a speaker was about 11 breaths per minute. Quantity of filled pauses in a minute of recordings was often surprisingly high, especially for inexperienced speakers (even above 10 per minute). Mean frequency of different types of pauses are compared in Table 3.

\subsection{Analysis of correlation of pauses and punctuation marks}

The information on frequency of using punctuation in spoken language was obtained by analyzing the quantity of full stops and commas in transcriptions. Figure 3 shows meaning of the pauses in determining punctuation in speech. Among all full stops in transcription, $39 \%$ are correlated with occurrences of a breath pause, $27 \%$ a silent pause, and $20 \%$ a filled pause (Fig 3a). Among all commas, $28 \%$ are pointed by a silent pause, $20 \%$ a breath pause, and $6 \%$ a filled pause (Fig 3b). Lack of any kind of a pause (words bonding in pronunciation) was registered in $20 \%$ occurrences of full stops and $46 \%$ commas for spontaneous speech and only for $1.3 \%$ full stops and $42 \%$ commas for read speech (Fig. 4). Among all occurrences of filled pauses, $8 \%$ indicate full stops and $6 \%$ indicate commas; among breath pauses the proportions are, respectively, 10 and $11 \%$ (Fig. 5).

However, the usage of different types of pauses for signalization of punctuation is strongly individualized between speakers, as presented in Tab. 4. To facilitate the observation of inter-speaker differences, the intensity of connection between pauses and punctuation was graded with grayscale. Although the general tendency was

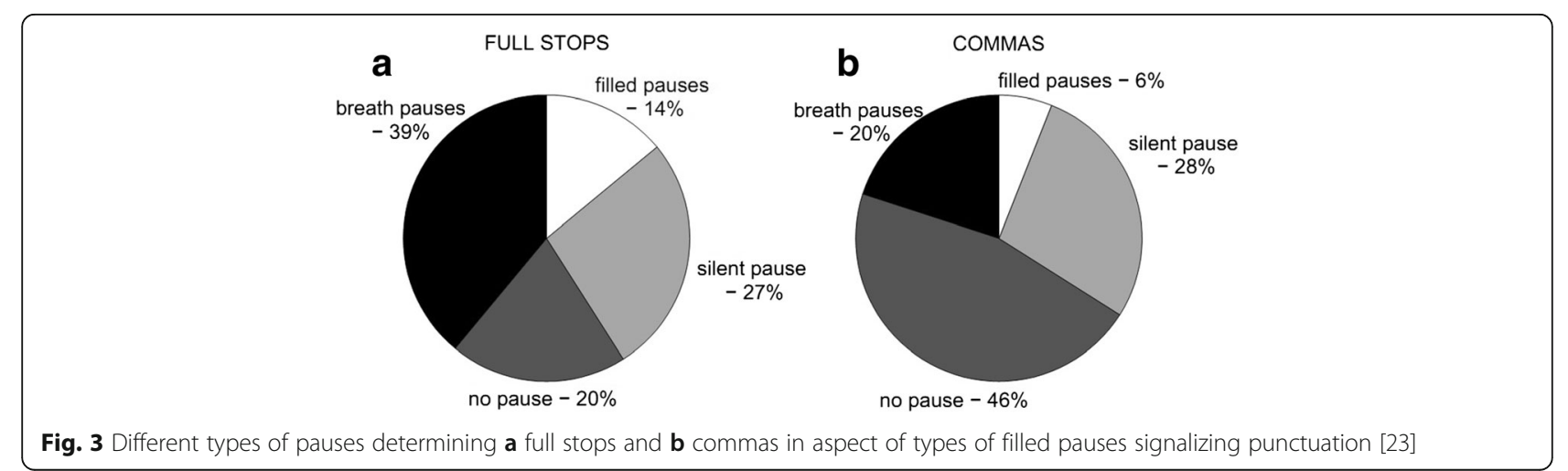




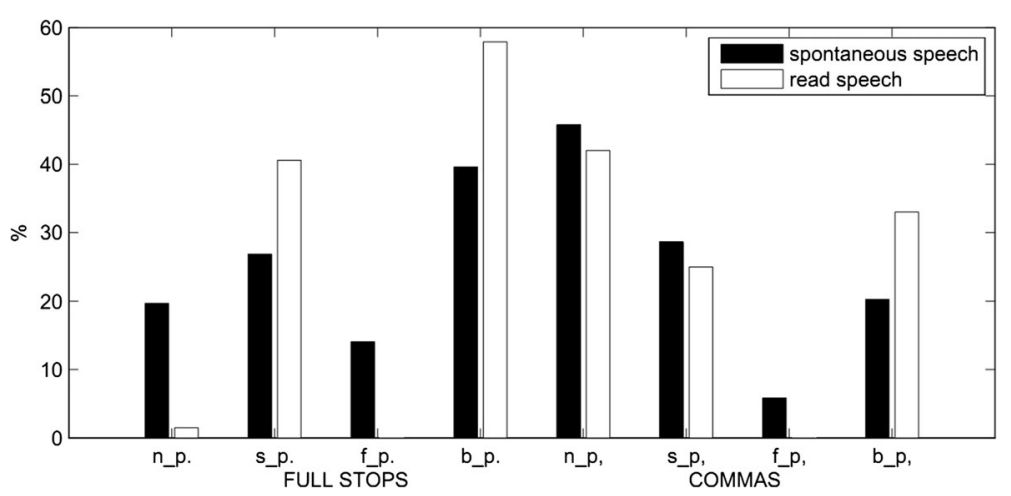

Fig. 4 Different types of pauses determining full stops and commas [23]

signalization of full stops with breaths and lack of any kind of a pause in the place of comma, the variation between speakers is considerable.

\subsection{Differences between speakers in quality and quantity of pauses}

Using feature vectors $p_{s}$ specific for each speaker $s$, we investigated correlation of each pair of speakers (formula 3 ). The obtained correlation matrix is presented in Fig. 6. Distribution of correlation of the given speaker with the others is illustrated in Fig. 7.

As it is presented in Figs. 5 and 6, speakers vectors were usually correlated to a small extent or not correlated. Speakers' distance from a mean vector was calculated according to formula (2). Distribution of results is presented in Fig. 8.

Having annotated a large quantity of breaths and filled pauses "yyy" for every speaker (with the latter not appearing only for 2 out of 30 speakers in our corpus), we decided to analyze inter-speaker differences. We observed that the durations of breaths in our corpus have mean $392 \mathrm{~ms}$, standard deviation $118 \mathrm{~ms}$, median
$368 \mathrm{~ms}$, and quantiles are as follows: 0.25 is $312 \mathrm{~ms}$ and 0.75 is $455 \mathrm{~ms}$. For the filled "yyy" pauses, the mean is $398 \mathrm{~ms}$, standard deviation is $183 \mathrm{~ms}$, median is $362 \mathrm{~ms}$, and quantiles are as follows: 0.25 is $278 \mathrm{~ms}$ and 0.75 is 484 ms. Analysis of variance showed, that both for breaths $(p=7.6 \mathrm{E}-50)$ and for filled "yyy" pauses $(p=$ $7.62 \mathrm{E}-22)$ the mean duration differences between speakers are statistically significant.

Figure 9a shows the results of ANOVA of breath duration of different speakers, and Fig. 9b shows the same for filled "yyy" pause duration. Analysis of those plots leads to the conclusion that although the differences are not statistically significant for every speaker pair, it makes sense to group the speakers into 2 or 3 categories, as in: speakers taking short breaths, speakers taking breaths of average length, speakers taking long breaths, and the same for "yyy" fillers. If more recordings of a single speaker were available, similar analysis could be made for the frequency of pause occurrence.

For the parameters of breaths and fillers duration, Gaussian models were created (in Fig. 10, an example

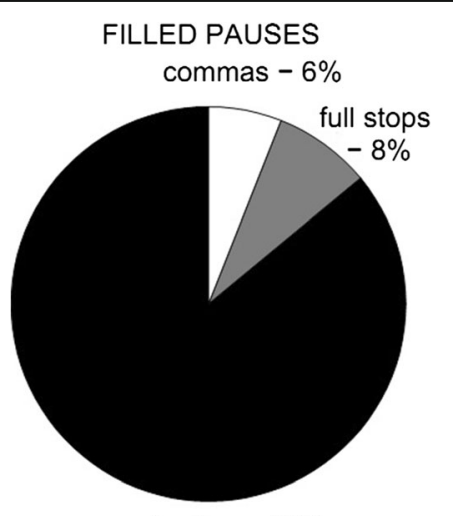

no punctuation - $86 \%$

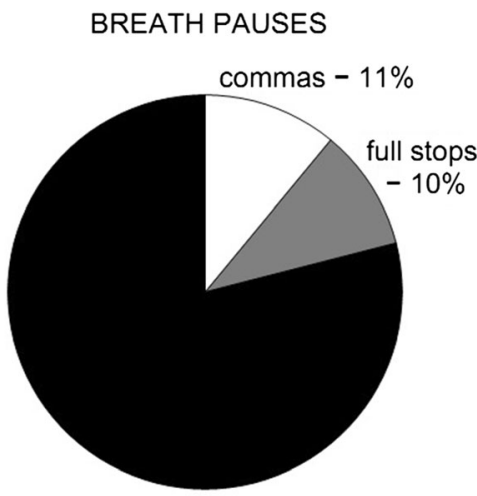

no punctuation $79 \%$

Fig. 5 Proportions of filled pauses and breath pauses occurrences correlated with full stops or commas [23] 
Table 4 Percent of pauses events denoting full stops and commas for each speaker ( $P$ presentations/orations, $T$ translation, $R$ radio interviews)

\begin{tabular}{|c|c|c|c|c|c|c|c|c|}
\hline \multirow[b]{2}{*}{ Recordings } & \multicolumn{4}{|c|}{ Full stops } & \multicolumn{4}{|c|}{ Commas } \\
\hline & n_p. & s_p. & f_p. & b_p. & n_p, & s_p, & f_p, & b_p, \\
\hline P1_5 & 12 & 65 & 24 & 0 & 38 & 49 & 18 & 0 \\
\hline P2_5 & 4 & 23 & 73 & 0 & 40 & 45 & 15 & 0 \\
\hline P3_3 & 7 & 93 & 0 & 0 & 38 & 63 & 0 & 0 \\
\hline P4_1 & 18 & 18 & 0 & 64 & 67 & 17 & 0 & 17 \\
\hline P5_2 & 40 & 0 & 20 & 40 & 64 & 9 & 0 & 27 \\
\hline P6_1 & 0 & 13 & 13 & 75 & 70 & 20 & 0 & 10 \\
\hline P7_1 & 0 & 25 & 25 & 50 & 43 & 21 & 7 & 29 \\
\hline P8_1 & 0 & 14 & 29 & 57 & 67 & 17 & 0 & 17 \\
\hline P9_1 & 0 & 0 & 67 & 33 & 64 & 18 & 18 & 0 \\
\hline P10_1 & 25 & 25 & 25 & 25 & 48 & 43 & 5 & 5 \\
\hline P11_5 & 3 & 45 & 0 & 52 & 12 & 22 & 0 & 67 \\
\hline P12_4 & 33 & 25 & 3 & 40 & 56 & 24 & 1 & 18 \\
\hline Type P & 14 & 34 & 20 & 32 & 44 & 34 & 7 & 16 \\
\hline T1_1 & 57 & 14 & 0 & 29 & 71 & 29 & 0 & 0 \\
\hline T2_1 & 25 & 25 & 25 & 25 & 57 & 0 & 14 & 29 \\
\hline T3_1 & 38 & 50 & 0 & 13 & 75 & 25 & 0 & 0 \\
\hline T4_1 & 0 & 45 & 0 & 55 & 38 & 13 & 0 & 50 \\
\hline T5_1 & 50 & 13 & 0 & 38 & 63 & 0 & 13 & 25 \\
\hline T6_5 & 19 & 13 & 16 & 53 & 51 & 23 & 2 & 25 \\
\hline T7_5 & 67 & 6 & 6 & 22 & 49 & 27 & 2 & 22 \\
\hline T8_5 & 0 & 25 & 4 & 71 & 28 & 28 & 4 & 40 \\
\hline T9_2 & 0 & 33 & 11 & 56 & 38 & 8 & 0 & 54 \\
\hline T10_4 & 30 & 19 & 15 & 37 & 61 & 18 & 2 & 18 \\
\hline T11_1 & 40 & 0 & 0 & 60 & 46 & 15 & 0 & 38 \\
\hline T12_2 & 18 & 12 & 0 & 71 & 32 & 16 & 16 & 37 \\
\hline Type T & 25 & 20 & 8 & 47 & 49 & 20 & 4 & 27 \\
\hline R1_10 & 47 & 14 & 3 & 36 & 63 & 20 & 2 & 15 \\
\hline R2_10 & 12 & 11 & 25 & 53 & 45 & 13 & 18 & 24 \\
\hline R3_10 & 36 & 4 & 22 & 39 & 69 & 4 & 14 & 14 \\
\hline R4_10 & 30 & 11 & 32 & 27 & 63 & 5 & 9 & 23 \\
\hline R5_10 & 33 & 16 & 41 & 10 & 61 & 17 & 11 & 12 \\
\hline R6_10 & 44 & 0 & 43 & 14 & 73 & 12 & 8 & 8 \\
\hline Type R & 34 & 9 & 17 & 40 & 62 & 12 & 13 & 13 \\
\hline All speakers & $23(20)$ & $22(21)$ & $17(19)$ & $38(22)$ & $53(15)$ & $21(14)$ & $6(7)$ & $21(17)$ \\
\hline
\end{tabular}

for nine speakers is presented). The preliminary analysis suggests that the features can be used for classification with Gaussian Mixture Models (GMM).

An interesting observation is that 8 (out of 30) of the speakers in our corpus do not use " $\mathrm{mmm}$ " filled pauses at all, while most of them do it rarely (on average about 1 per minute, compared to almost 7 filled "yyy" pauses per minute). The most frequent use of them is made by speaker T9_2 at about 3.5 "mmm" pause per minute. This leads to a conclusion that frequent usage of " $\mathrm{mmm}$ " filled pause is a characteristic feature of a speaker.

In the next experiment, standardized values of each feature were quantized into three values: low, medium and high, in reference to the distribution of each feature. For the quantized feature matrix (Fig. 11a), a clusterization was performed, using Euclidean distance measure. The obtained dendrograms with a heatmap representation 

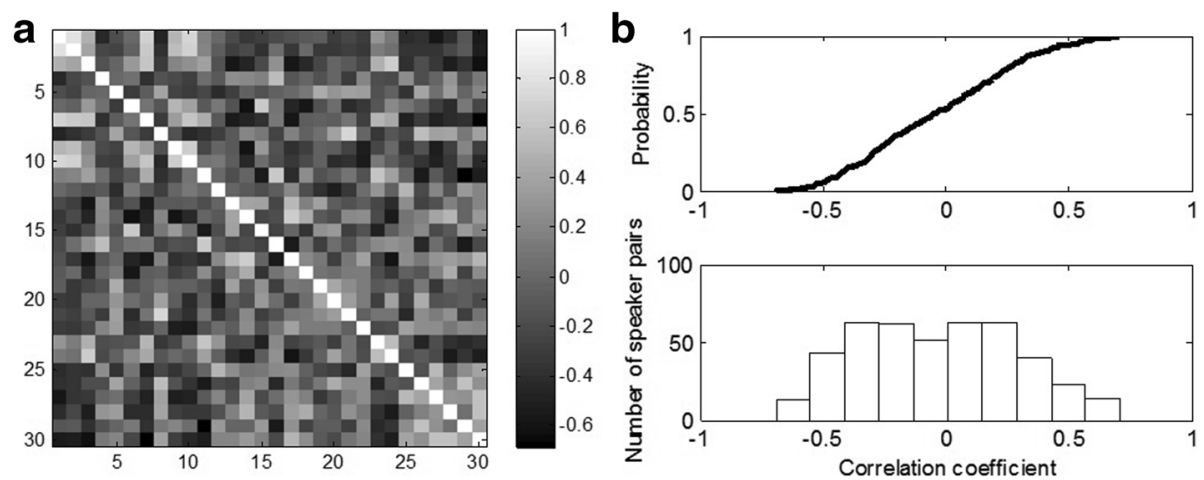

Fig. 6 a Correlation matrix for 30 speakers. b Cumulative distribution function and histogram of correlation coefficients for pairs of speakers

allow to easily observe in which features the most similar speakers were alike.

\subsection{Group analysis}

To investigate the influence of experience and oratorical abilities on pauses and speech rate, we divided a corpus of spontaneous monologues into recordings of experienced speakers (professors and politicians) and inexperienced speakers (mainly students). Average values of selected temporal features of each group are compared in Table 5.

As expected intuitively, professionals speak more slowly, with less disfluencies and formulate shorter sentences, which makes their speech more adjusted for efficient listening and understanding by recipients. Also their dynamic breathing rhythms are much more concordant with sentences boundaries (a half of full stops were correlated with breath pauses). Such conscious dynamic breathing (taking a breath before beginning of a sentence or phrase) is one of the basic voice emission principles, often emphasized by authors of handbooks on speaking skills and techniques.

The comparison of presentations, translations and radio dialogues can be observed in Fig. 12. Some of differences are significant and can be interpreted in accordance to intuitive situational context conditions. In radio interviews speakers tend to speak much faster, which is conditioned by the determined time for the conversation. Surprisingly, their filled pauses are much longer than for the rest of analyzed recordings.

\subsection{Using pauses for speaker recognition-evaluation results}

For the experiment, recordings from audiobooks were used (50 speakers, $15 \mathrm{~min}$ for each speaker). The choice was made in order to obtain a regular set of long enough recordings in the same situational context (reading a story) from a similar group of speakers (professional lectors). Entire set was used to train UBM and Total Variability matrix in the i-vector system.

Recordings were split into train part of $5 \mathrm{~min}$ and four test parts of $2.5 \mathrm{~min}$ each. It allowed performing 1200 cross-validation tests: 200 verification of authorized users (target trials) and 1000 simulation of impostors attacks (10 impostors with 2 random recordings were chosen randomly for each speaker). Parameters of silent pauses $\left(f_{1}-f_{21}\right)$ were automatically extracted with VAD algorithm and the feature sets were processed as independent stream (as presented in Fig. 1) according to the methodology described in chapter 3.4.

The results of speaker verification task of a baseline i-vector system (using only MFCC features) are presented in Fig. 13, where the red and blue histograms are normalized distributions of target and impostor scores respectively. Vertical axis refers to FNR and FPR

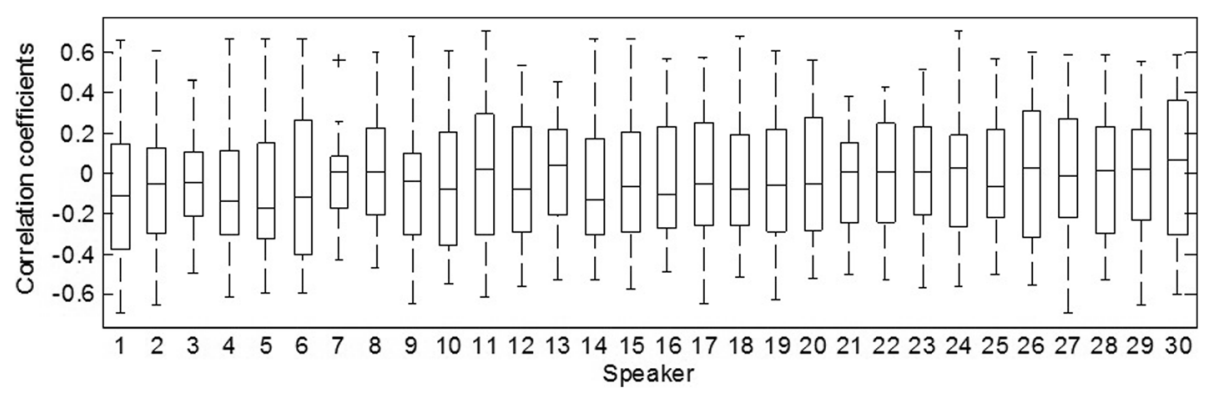

Fig. 7 Boxplot for correlation coefficients distribution for each speaker 


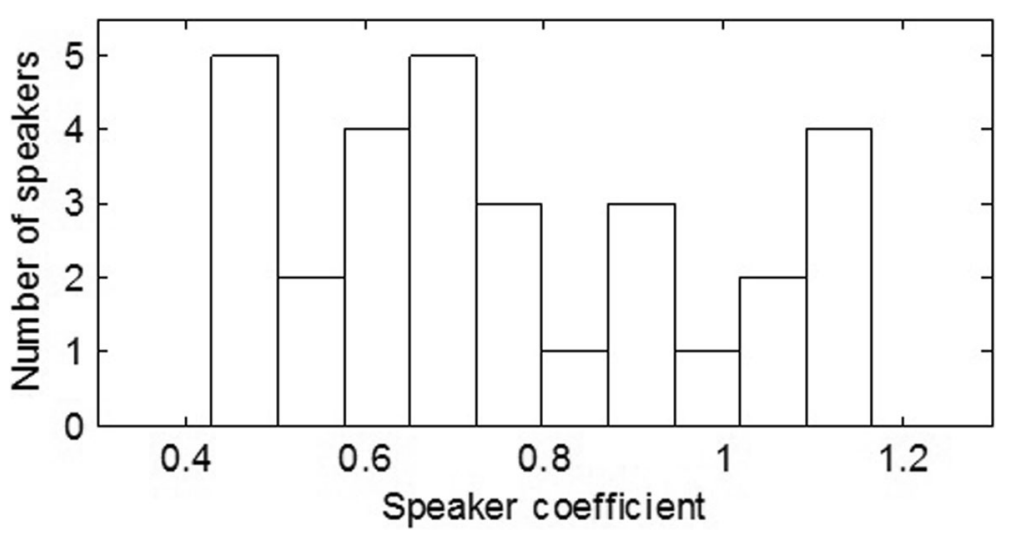

Fig. 8 Histogram of coefficients $\gamma_{s}$ for all speakers

values. This result was obtained for the system based on 1024-component GMM-UBM and 400-dimensional total variability subspace. In this case EER reached the smallest value-3 $\%$. The result is considered as sound for the number of impostor and target speakers used in the test. The performance of the system might be enhanced by gender-dependent approach or incorporation of score processing like PLDA or score normalization techniques, but the goal of this experiment was measuring efficacy of a simple baseline speaker verification system on the dataset.

Performance of the system based on pauses features is illustrated in Fig 14. For the evaluation three features were used: durations of the silent pauses, number of silent pauses per minute $\left(f_{1}\right)$ and ratio between sum of pause duration $\left(f_{2}\right)$ to entire signal length. Due to limited number of data points that was extracted from test samples it was necessary to use minimal number of components in GMM to prevent overfitting. Best results were obtained with 4-component GMM. Such configuration resulted in EER equal to $40 \%$. Overlapped distributions in Fig 14 suggest that used features are little discriminative in speaker comparison. This meager result implies that applied features, without any further processing, should not be used as a standalone input into GMM classifier in a speaker discrimination task.

Figure 15 presents DET plots for Pauses and i-vectors systems and for the fusion of the systems performed with a Bosaris Toolkit, where $20 \%$ of the result scores were used to train fusion algorithm. Value of $20 \%$ were chosen empirically, as an optimal point for DET plot. Modification of this value by enlarging training dataset did not change the positions of the curve but reduced its resolution due to lower number of test points. The fusion of the scores of the two systems caused no gain in overall performance and reveals reduction of efficacy by $1 \%$ in terms of EER.

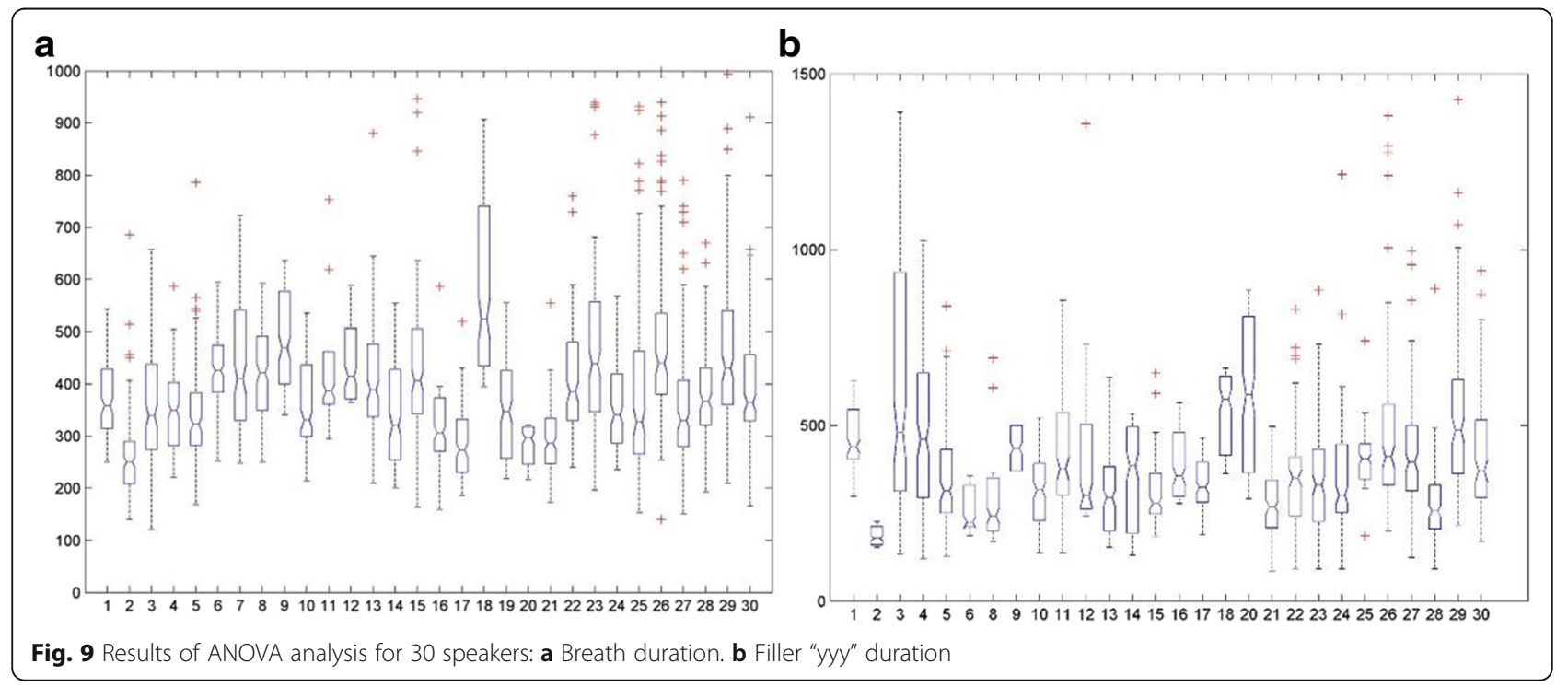



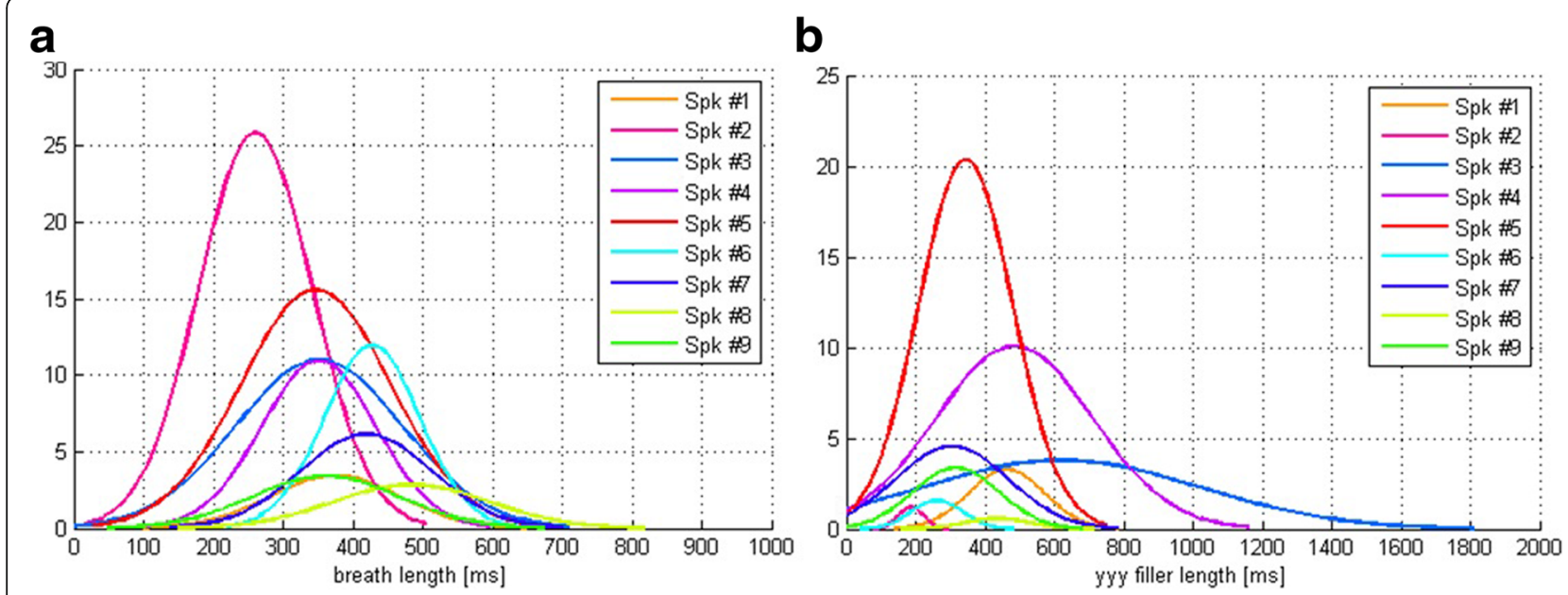

Fig. 10 Gaussian models fitted for nine speakers for a breath duration and $\mathbf{b}$ filler "yyy" duration

4.6 Automatic recognition of type of spontaneous speech For this task, as our features we used a time series of silent pause information extracted in an online manner, where each point indicates an appearance of a silent pause. Three features were used in the experiment: the duration of the silent pause instance, s_p per minute $\left(f_{1}\right)$ and percentage of s_p time in recording $\left(f_{2}\right)$, where $f_{1}$ and $f_{2}$ were calculated online, based on the silent pause instances gathered up to this point. To perform classification, we adopted a diagonal covariance GMM classifier with three mixtures (one for each class of spontaneous speech). The best

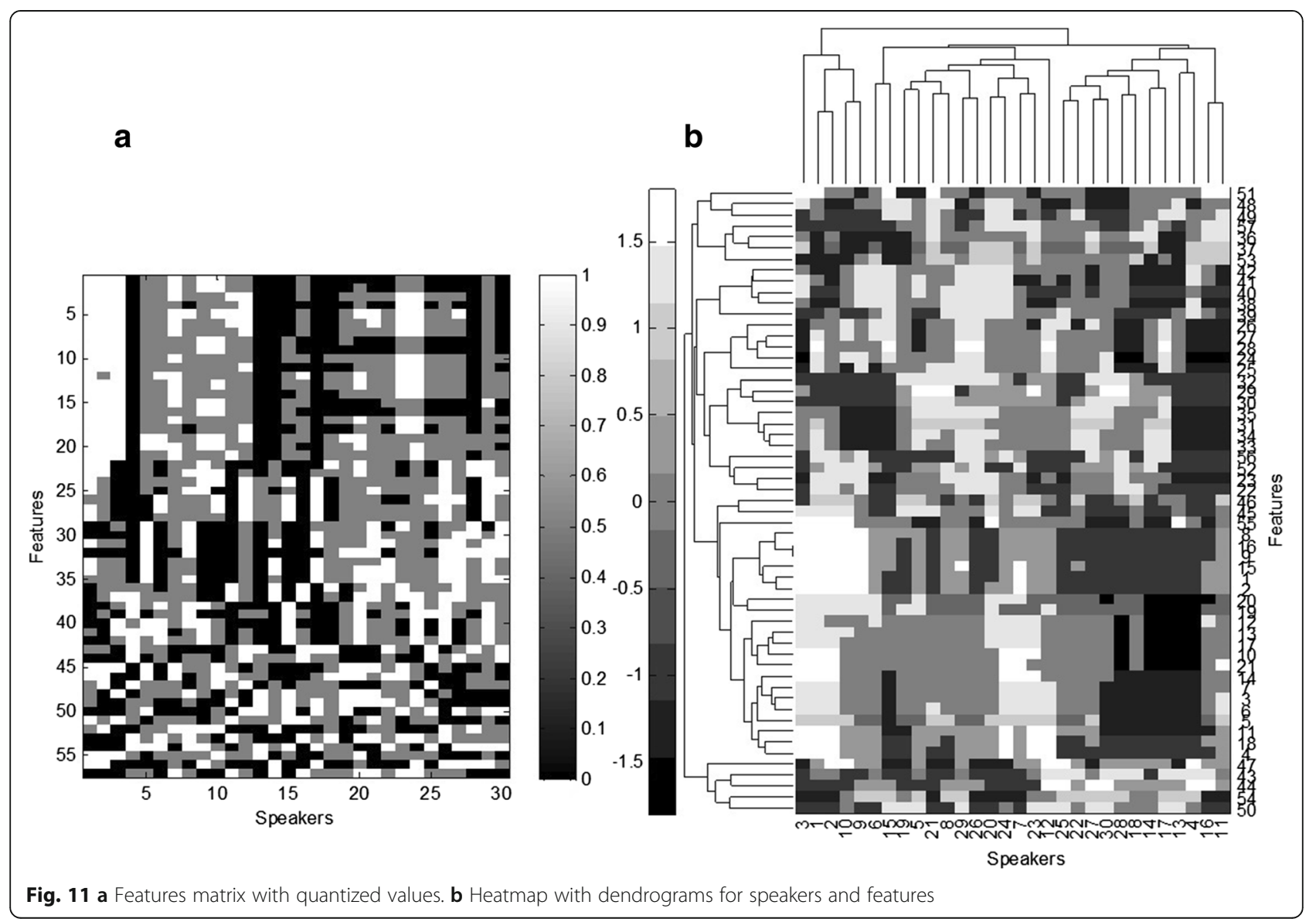


Table 5 Comparison of selected features for experienced and inexperienced speakers: average values and standard deviation (in brackets)

\begin{tabular}{lll}
\hline Parameter & Professional speakers & Inexperienced speakers \\
\hline \#words/minute & $108(23)$ & $117(26)$ \\
\#words/sentence & $17(4)$ & $22(6)$ \\
\#f_p/minute & $4(4)$ & $10(5)$ \\
n_p. [\%] & $12(15)$ & $13(13)$ \\
s_p. [\%] & $26(31)$ & $24(23)$ \\
f_p. [\%] & $10(12)$ & $34(30)$ \\
b_p. [\%] & $50(17)$ & $27(8)$ \\
\hline
\end{tabular}

result was achieved for three Gaussian components in each mixture. Experiment was carried out using a "leave-one-out" cross-validation scheme.

The classifier achieved $78 \%$ accuracy. Table 6 shows the Precision, Recall and F1 score achieved by the classifier in this task. As illustrated by the confusion matrix in Fig. 16, the worst performing class is the T type (translations), as it tends to be mistaken as a P type (presentation/oration). We suspect this is due to speakers making long silent pauses in both scenarios-to make a rhetoric effect in case of P, and because of the necessity to wait for more context before translating an utterance in case of $\mathrm{T}$.

\subsection{Automatic classification of read and spontaneous speech}

In this task, we confronted our spontaneous speech recordings ( $\mathrm{P}, \mathrm{T}$, and $\mathrm{R}$ classes) with 50 audiobook recordings (A class). In the distinction between read and spontaneous speech, seven features $\left(f_{1}-f_{7}\right)$ describing silent pauses were used. This choice was due to presence of this type of pauses in any recording (in contrast to filled pauses which are absent in read speech and breath pauses which are present only in good quality recordings) and easiness of detecting them automatically. The silent pauses were found automatically using VAD and then, for each recording, a vector of seven features was calculated. Logarithmization and normalization of the parameters improved the results.

The best accuracy in this task was obtained using XGBoost classifier (Table 7). However, it should be noted that the dataset is imbalanced in terms of classes, and this classifier exhibits bias toward the read speech class which shows as increasing read speech recall and decreasing spontaneous speech recall in regard to less complex classifiers such as the decision tree. Nonetheless, all classifiers perform better than if classification was done by chance (50\% accuracy), or by always indicating the class with higher count (65\% accuracy).

We observed that read speech was better recognizable than spontaneous speech (see Fig. 17), which we believe is partially a result of classification bias, but also a result of the higher diversity of spontaneous speech class examples.

\section{Discussion}

Majority of speaker recognition systems do not include suprasegmentals. High-level features of speech signal, like pauses, although statistically they were proved to be speaker-specific, are dependent also on other factors, like situational context, stress level, kind of linguistic task. This weakens their possible usage for speaker recognition. It should also be remembered that obtaining data on pauses requires much longer (than e.g. standard MFCC analysis) segment of continuous speech to perform analysis, which is not a desirable situation in a text-dependent system which operate on short utterances (e.g. one sentence). Relatively large observation period (at least one minute) sufficient for acquiring information on pausing style, constraints applying the approach only to certain sort of 


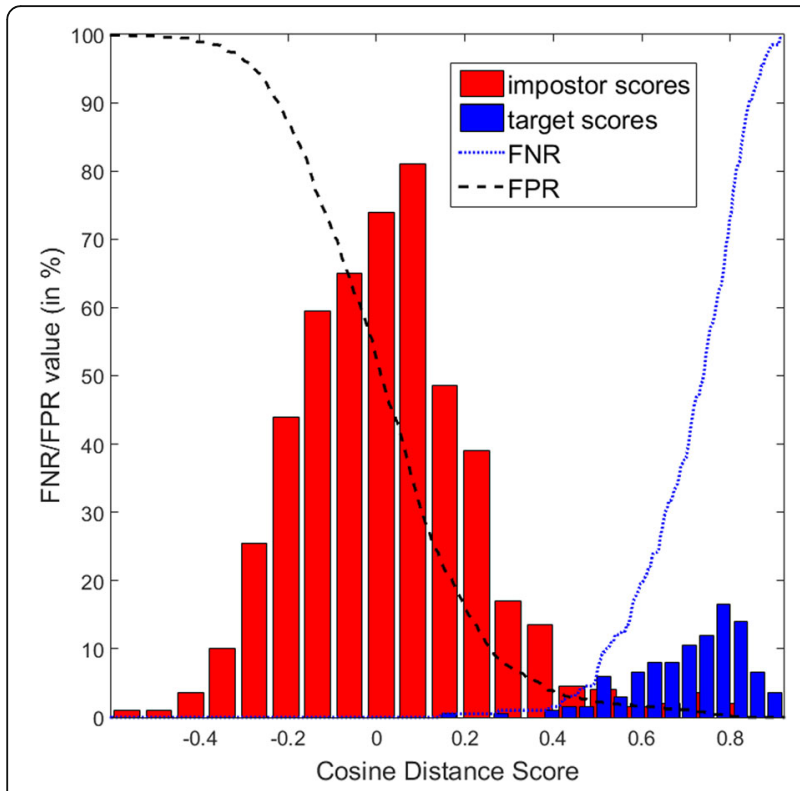

Fig. 13 Performance of the baseline system

biometric systems (text-independent systems or forensic applications).

The results obtained from testing a system based on pauses only (40\%) are similar to EER achieved in Peskin et al. test (36.1 to $43.3 \%$ EER) using different pause-related features alone [16]. However, more types of models and classifiers should be tested in future works. Probably pauses would be better included into model using HMM chain, as applied in [25] or as a part of n-gram models [26].

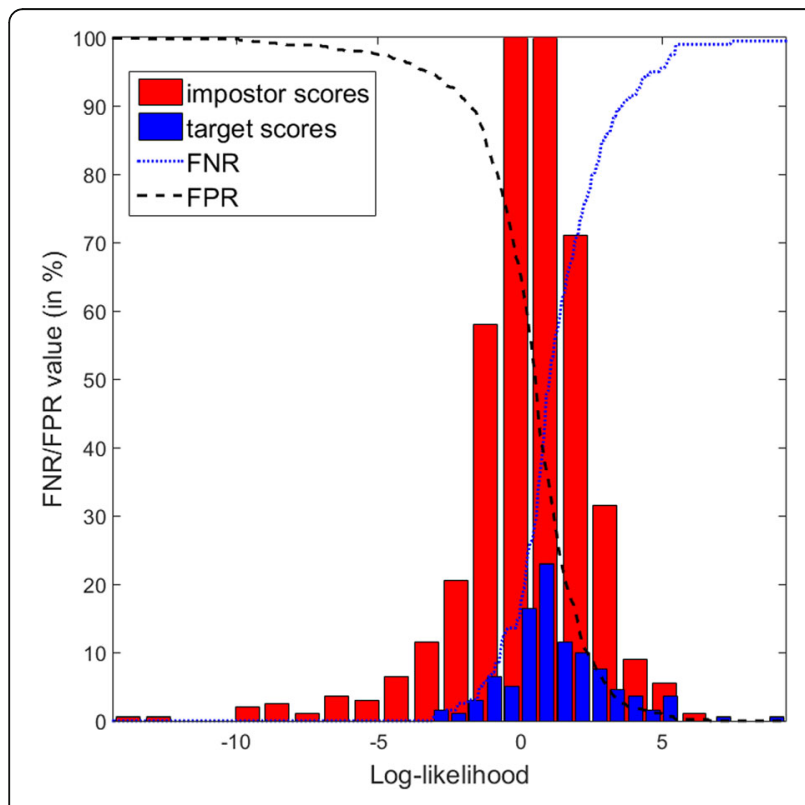

Fig. 14 Performance of system based on pause-related features only

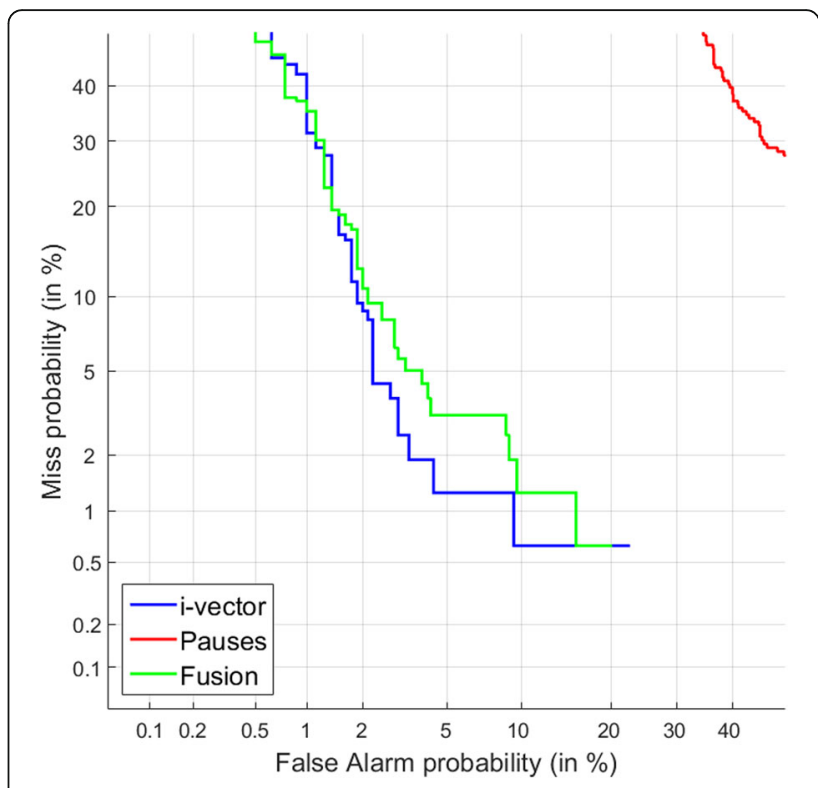

Fig. 15 Detection Error Trade-off plot for pause-based and MFCC-based systems and their fusion.

This data is not enough in itself to perform biometric verification or identification of a speaker; however, it can be used to enhance speech technology applications by including additional information in speaker's profile, such as the following: speaker breathes frequently, takes short breaths, makes filled pauses infrequently, etc.

In the second experiment, it was showed that the distribution and structure of pauses in speech, represented by three parameters, are specific for the type of speech and sufficient to automatically classify them with $78 \%$ accuracy. We showed that parameters such as a number of each pause type occurrences per minute or statistics of pause duration bring important information about speaker's habits. Advantage of the approach is simplicity, low computational complexity and robust feature extraction. Breath events [2] and filled pauses [23] can be automatically detected in a speech signal. It allows including the features easily in speech technology systems.

The obtained knowledge on pauses meaning can be merged with analysis of other temporal features (phoneme length, energy, fundamental frequency) in order to build algorithms for punctuation detection in speech. Since lack of punctuation and occurrence of disfluencies in

Table 6 Results of automatic recognition of types of spontaneous speech

\begin{tabular}{lllll}
\hline Type & Precision & Recall & F1-score & Support \\
\hline $\mathrm{P}$ & 0.71 & 0.91 & 0.80 & 11 \\
$\mathrm{~T}$ & 0.78 & 0.70 & 0.74 & 10 \\
$\mathrm{R}$ & 1.00 & 0.67 & 0.80 & 6 \\
Average/ total & 0.80 & 0.78 & 0.78 & 27 \\
\hline
\end{tabular}




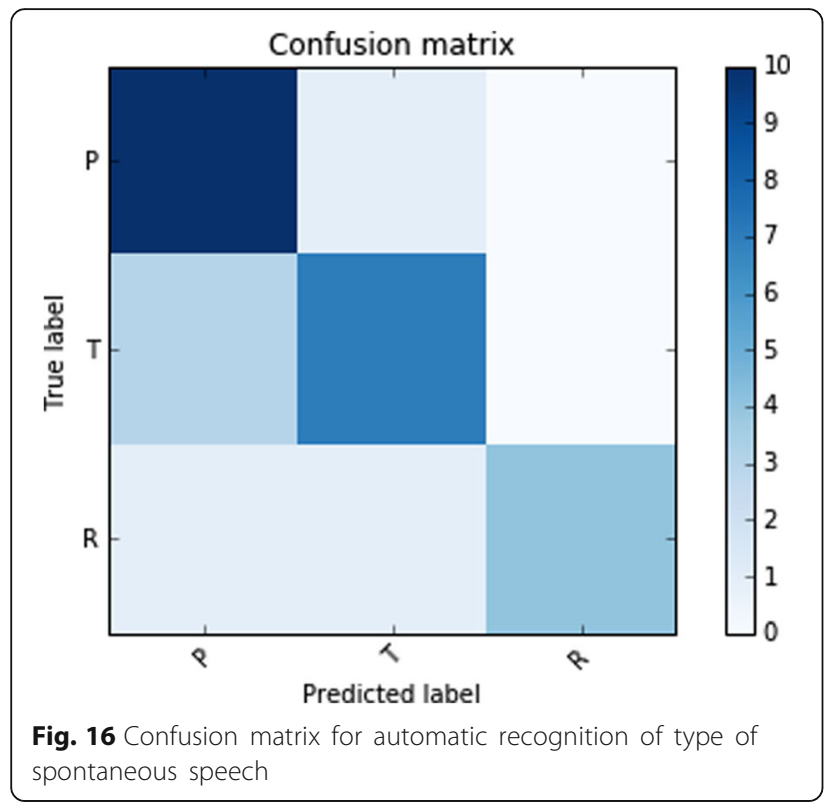

spontaneous speech transcripts are factors that disturb their processing by natural language processing systems, parsers or information extraction systems, automatic analysis of pauses can help to make spontaneous speech transcripts more readable for both human and NLP systems.

Finally, in the biomedical field, the research on pauses is meaningful in affect detection. All analyzed kinds of pauses carry information on speaker current emotional state. Frequency and regularity of pausing behavior, based on obtained models, is currently tested in the task of automatic emotion recognition. It can lead directly to include it in

Table 7 Results of automatic recognition of read and spontaneous speech - comparison of classifiers

\begin{tabular}{llllll}
\hline Classifier & Type & Precision & Recall & F1 score & Accuracy \\
\hline Decision tree & Read & 0.75 & 0.78 & 0.76 & 0.69 \\
& Spontaneous & 0.56 & 0.52 & 0.54 & \\
& Average & 0.68 & 0.69 & 0.69 & \\
Logistic regression & Read & 0.74 & 0.86 & 0.80 & 0.71 \\
& Spontaneous & 0.63 & 0.44 & 0.52 & \\
& Average & 0.70 & 0.71 & 0.70 & \\
SVM (RBF kernel) & Read & 0.75 & 0.88 & 0.81 & 0.73 \\
& Spontaneous & 0.67 & 0.44 & 0.53 & \\
& Average & 0.72 & 0.73 & 0.71 & \\
Random forest & Read & 0.73 & 0.86 & 0.79 & 0.70 \\
& Spontaneous & 0.61 & 0.41 & 0.49 & \\
& Average & 0.69 & 0.70 & 0.68 & \\
XGBoost & Read & 0.75 & 0.94 & 0.83 & 0.75 \\
& Spontaneous & 0.79 & 0.41 & 0.54 & \\
& Average & 0.76 & 0.75 & 0.73 & \\
\hline
\end{tabular}

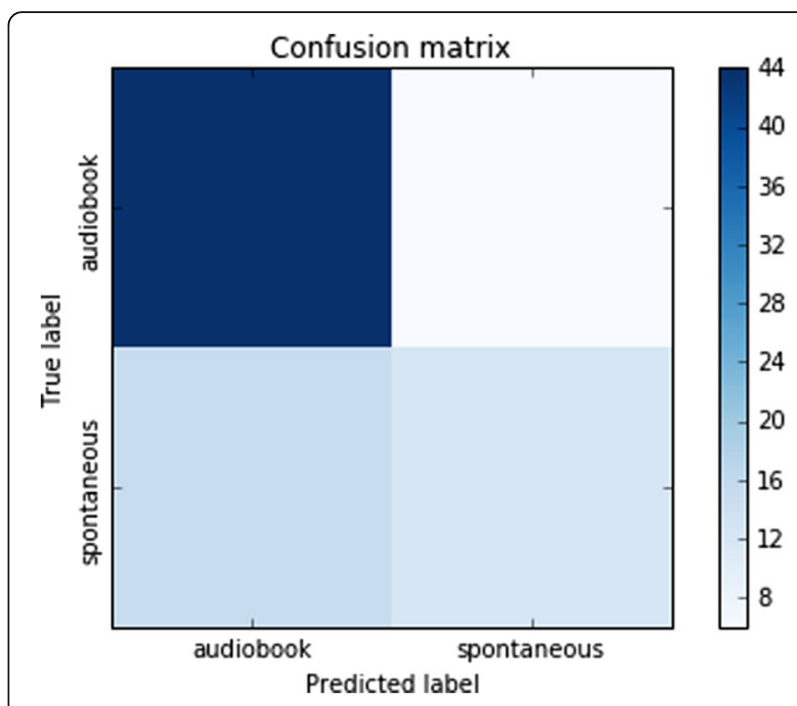

Fig. 17 Confusion matrix for automatic recognition of $\mathrm{read} / \mathrm{spontaneous} \mathrm{speech}$

systems for monitoring mental illnesses, since quantity and duration of silent pauses can be indicators of emotional state of the speaker or a measurable symptom of psychic disorders like schizophrenia or bipolar affective disorders. Measuring breath frequency in acoustic signal can be a cheap and easily available method for estimation of physical effort level, measure of physical fitness or diagnostics of potential respiratory dysfunctions (e.g. sleep apnea).

\section{Conclusions}

In this paper, we deliver the numerical description of pauses in Polish speech. Three types of acoustic pauses (silence, breaths and fillers), two types of punctuation marks (full stops and commas), and co-occurrences of acoustic and syntactic pauses were proved to be speaker dependent. Pausing behavior was investigated in several contexts (spontaneous speech during presentation, simultaneous interpretation, interview and read speech reading a novel).

Connotations between pauses and punctuation, as well as frequency and types of pauses vary between individuals and depend on speaking style of each person, speech quality, culture, experience and preparation for oral presentations. Thereby, the temporal features can possibly be used as a valuable source of paralinguistic information. However, even though our results were better than similar previous studies, the differences were not sufficient to differentiate speakers. Verification of the hypothesis that they improve speaker recognition system was negative for scenario of modeling pauses with UBM and GMM models. Another modeling methods will be evaluated in future works.

An attempt to automatically recognize three types of spontaneous speech resulted in $78 \%$ accuracy and distinguishing read and spontaneous speech with $75 \%$ accuracy, 
using pause-related features only. This result shows usefulness of pauses to distinguish between different situational context and cognitive task and therefore it could find application for automatic discourse analysis and conversation modeling purposes. Presented statistical models of pauses will be a fundament for studying usefulness of the information in different applications, like ASR or emotion recognition systems. Further research will cover also other reasons of pauses frequency and duration variability (a type of personality of the speaker and emotional arousal). Feature vector dimensionality will be reduced. Analyses will be also conducted on more regular sets of recordings, e.g., the same speaker in different situational contexts.

\section{Acknowledgements}

The project was supported by The Polish National Centre for Research and Development granted by decision 072/R/ID1/2013/03 and the National Science Centre support allocated on the basis of a decision DEC-2011/03/D/ST6/00914. This work was supported by the AGH University of Science and Technology under Deans Grant 15.11.230.252 and Statutory works 11.11.230.017.

\section{Competing interests}

The authors declare that they have no competing interests.

\section{Author details}

'Department of Computer Science, Electronics and Telecommunications, AGH University of Science and Technology, Al. Adama Mickiewicza 30, 30-059 Kraków, Poland. Techmo, Kraków, Poland.

\section{Received: 28 November 2015 Accepted: 28 October 2016}

Published online: 09 November 2016

\section{References}

1. F Batista, H Moniz, I Trancoso, N Mamede, A Mata, Extending automatic transcripts in a unified data representation towards a prosodic-based metadata annotation and evaluation. Journal of Speech Sciences 2(2), 115-138 (2012)

2. M Igras, B Ziółko: Wavelet method for breath detection in audio signals. In: IEEE International Conference on Multimedia and Expo (ICME 2013), San Jose (2013). doi:10.1109//CME.2013.6607428

3. TKendall, Speech rate, pause and linguistic variation: an examination through the sociolinguistic archive and analysis project. Doctoral dissertation (Duke University, Durham, 2009)

4. E Campione, J Véronis.(2002). A large-scale multilingual study of silent pause duration. In: Proceedings of the Speech Prosody Conference, 199-202

5. M Demol, W Verhelst, P Verhoeve. (2006). A study of speech pauses for multilingual time-scaling applications. In: Proc. ISCA-ITRW Multiling, (Stellenbosch, South Africa).

6. I Homma, Y Masaoka, Breathing rhythms and emotions. Experimental physiology 93(9), 1011-1021 (2008)

7. American Thoracic Society and American College of Chest Physicians, ATS/ ACCP Statement on cardiopulmonary exercise testing. American Journal of Respiratory and Critical Care Medicine 167(2), 211-277 (2003)

8. V Rapcan, S D'Arcy, S Yeap, N Afzal, J Thakore, RB Reilly, Acoustic and temporal analysis of speech: a potential biomarker for schizophrenia. Medical Engineering \& Physics 32, 1074-1079 (2010)

9. D Baron, E Shriberg, A Stolcke. (2002). Automatic punctuation and disfluency detection in multi-party meetings using prosodic and lexical cues. In: Proceedings of the International Conference on Spoken Language Processing, 949-952

10. E Shriberg, A Stolcke, D Hakkani- Tür, G Tür, Prosody-based automatic segmentation of speech into sentences and topics. Journal Speech Communication - Special issue on accessing information in spoken audio archive 32(1-2), 127-154 (2000)

11. WA Lea, Trends in speech recognition (Academic Press, New York, 1980)

12. V Ramanarayanan, E Bresch, D Byrd, L Goldstein, SS Narayanan, Analysis of pausing behavior in spontaneous speech using real-time magnetic resonance imaging of articulation. The Journal of the Acoustical Society of America 126, 160-165 (2009)

13. T Kinnunen, $\mathrm{H} L \mathrm{~L}$, An overview of text-independent speaker recognition: from features to supervectors. Speech communication 52(1), 12-40 (2010)

14. B Ziółko, W Kozłowski, M Ziółko, R Samborski, D Sierra, J Gałka, Hybrid wavelet-Fourier-HMM speaker recognition. International Journal of Hybrid Information Technology 4(4), 25-41 (2011)

15. E Shriberg, Higher-level features in speaker recognition. Speaker Classification I. Lecture Notes in Computer Science / Artificial Intelligence (Springer, Berlin/ Heidelberg, 2007), pp. 241-259

16. B Peskin, J Navratil, J Abramson, D Klusacek, DA Reynolds, X Bing: Using prosodic and conversational features for high-performance speaker recognition: report from JHU WS'02. IEEE International Conference on Acoustics, Speech, and Signal Processing (2003). doi: 10.1109/ICASSP.2003.1202762

17. K Sönmez, E Shriberg, L Heck, M Weintraub. (1998). Modeling dynamic prosodic variation for speaker verification. In: Proc. ICSLP, 3189-3192

18. G Adami, Modeling prosodic differences for speaker recognition. Speech Communication 49(4), 277-291 (2007)

19. M Backes, G Doychev, M Dürmuth, B Köpf. (2010). Speaker recognition in encrypted voice streams. In: Proceedings of the 15th European Conference on Research in Computer Security, 508-523

20. J Lööf, C Gollan, H Ney. (2009). Cross-language bootstrapping for unsupervised acoustic model training: rapid development of a Polish speech recognition system. In: Proceedings of Interspeech, Brighton, 88-91

21. DA Reynolds, TF Quatieri, RB Dunn, Speaker verification using adapted Gaussian mixture models. Digital Signal Processing 10(1-3), 19-41 (2000)

22. J Pelecanos, S Sridharan: Feature warping for robust speaker verification. In: Proc. Speaker Odyssey: the Speaker Recognition Workshop (Odyssey 2001), Crete, Greece, 213-218 (2001)

23. M Igras, B Ziółko, Different types of pauses as a source of information for biometry. Models and analysis of vocal emissions for biomedical applications: 8th international workshop (Firenze University Press, Firenze, 2013), pp. 197-200

24. K Barczewska, M Igras, Detection of disfluencies in speech signal. Challenges of modern technology 32(1-2), 3-10 (2013)

25. F Beritelli, A Spadaccini. (2012). Performance evaluation of automatic speaker recognition techniques for forensic applications. New Trends and Developments in Biometrics, 129-148

26. E Shriberg, L Ferrer, S Kajarekar, A Venkataraman, A Stolcke, Modeling prosodic feature sequences for speaker recognition. Speech Communication 46(3), 455-472 (2005)

27. B Zellner, Pauses and the temporal structure of speech, in Fundamentals of speech synthesis and speech recognition, ed. by E Keller (Wiley, Chichester, 1994), pp. 41-62

28. E Shriberg: Spontaneous speech: How people really talk and why engineers should care. Proceedings of European Conference on Speech Communication and Technology, Eurospeech, 1781-1784 (2005)

29. B. Ziółko, T. Jadczyk, D. Skurzok, P. Żelasko, J. Gałka, T. Pędzimąż, I. Gawlik, S. Pałka .2015. "SARMATA 2.0 Automatic Polish Language Speech Recognition System", Interspeech, Dresden,

30. P Kenny. (2012). A small footprint i-vector extractor. Odyssey 2012: 1-6

31. https://sites.google.com/site/bosaristoolkit/ Accessed: 30 May 2016

32. R Dufour, Y Estève, P Deléglise, Characterizing and detecting spontaneous speech: application to speaker role recognition. Speech Communication 56, $1-18(2014)$

33. A Tóth, Speech disfluencies in simultaneous interpreting: a mirror on cognitive processes. SKASE Journal of Translation and Interpretation 5(2), 23-31 (2011)

34. B Tissi, Silent pauses and disfluencies in simultaneous interpretation: a descriptive analysis. The Interpreters' Newsletter 10, 103-127 (2000)

35. L Ten Bosch, N. Oostdijk, J P De Ruiter. (2004). Turn-taking in social talk dialogues: temporal, formal and functional aspects. In 9th International Conference Speech and Computer (SPECOM'2004). 454-461

36. J H Friedman, Greedy function approximation: a gradient boosting machine. Annals of statistics, 29(5), 1189-1232 (2001)

37. Pedregosa et al. (2011). Scikit-learn: Machine Learning in Python, JMLR 12, pp. $2825-2830$

38. P Żelasko, B Ziółko, T Jadczyk, D Skurzok, "AGH Corpus of Polish Speech". Language Resources and Evaluation 50, 585-601 (2016)

39. A Martin, G Doddington, T Kamm, M Ordowski, M Przybocki, "The DET curve in assessment of detection task performance", in Proceedings of the 5th European Conference on Speech Communication and Technology (Greece, EUROSPEECH, Rhodes, 1997). pp. 1895-1898 